\title{
Neuro-musculoskeletal simulation of instrumented contracture and spasticity assessment in children with cerebral palsy
}

\author{
Marjolein Margaretha van der Krogt ${ }^{1 *}$, Lynn Bar-On ${ }^{1,2,3}$, Thalia Kindt ${ }^{3}$, Kaat Desloovere ${ }^{2,3}$ and Jaap Harlaar ${ }^{1}$
}

\begin{abstract}
Background: Increased resistance in muscles and joints is an important phenomenon in patients with cerebral palsy (CP), and is caused by a combination of neural (e.g. spasticity) and non-neural (e.g. contracture) components. The aim of this study was to simulate instrumented, clinical assessment of the hamstring muscles in CP using a conceptual model of contracture and spasticity, and to determine to what extent contracture can be explained by altered passive muscle stiffness, and spasticity by (purely) velocity-dependent stretch reflex.

Methods: Instrumented hamstrings spasticity assessment was performed on 11 children with CP and 9 typically developing children. In this test, the knee was passively stretched at slow and fast speed, and knee angle, applied forces and EMG were measured. A dedicated OpenSim model was created with motion and muscles around the knee only. Contracture was modeled by optimizing the passive muscle stiffness parameters of vasti and hamstrings, based on slow stretch data. Spasticity was modeled using a velocity-dependent feedback controller, with threshold values derived from experimental data and gain values optimized for individual subjects. Forward dynamic simulations were performed to predict muscle behavior during slow and fast passive stretches.

Results: Both slow and fast stretch data could be successfully simulated by including subject-specific levels of contracture and, for CP fast stretches, spasticity. The RMS errors of predicted knee motion in CP were $1.1 \pm 0.9^{\circ}$ for slow and $5.9 \pm 2.1^{\circ}$ for fast stretches. CP hamstrings were found to be stiffer compared with TD, and both hamstrings and vasti were more compliant than the original generic model, except for the CP hamstrings. The purely velocity-dependent spasticity model could predict response during fast passive stretch in terms of predicted knee angle, muscle activity, and fiber length and velocity. Only sustained muscle activity, independent of velocity, was not predicted by our model.
\end{abstract}

Conclusion: The presented individually tunable, conceptual model for contracture and spasticity could explain most of the hamstring muscle behavior during slow and fast passive stretch. Future research should attempt to apply the model to study the effects of spasticity and contracture during dynamic tasks such as gait.

Keywords: Muscle spasticity, Muscle stiffness, Neuro-musculoskeletal modeling, Cerebral palsy, Biomechanics, Electromyography, Rehabilitation

\footnotetext{
* Correspondence: m.vanderkrogt@vumc.nl

'Department of Rehabilitation Medicine, VU University Medical Center, MOVE

Research Institute Amsterdam, PO Box 7057, 1007 MB Amsterdam, The

Netherlands

Full list of author information is available at the end of the article
} 


\section{Background}

Cerebral palsy $(\mathrm{CP})$ is the most common neurological disorder in children and is attributed to non-progressive disturbances occurring in the developing fetal or infant brain [1]. It is characterized primarily by neural deficits (caused by the brain anomalies) and secondary by muscular and bone deformities [2, 3]. These deficits adversely affect normal development of functional activities, such as gait. Muscle hyper-resistance, i.e. increased tension in a passively stretched muscle, caused by neural impairments (spasticity and increased background activation) as well as by passive tissue stiffness, is the most prevalent problem. Spasticity is commonly defined as a velocity-dependent increase in tonic stretch reflexes due to hyper-excitability [4], while stiffness is a mechanical resistance of the myotendinous tissue as it is passively lengthened [5].

The exact pathophysiology of hyper-resistance is complex and only partially understood. Specifically, there is lack of consensus on the mechanisms of, and interaction between, the neural and non-neural components of hyper-resistance. For example, it is generally thought that muscle stiffness occurs secondary to spasticity, with prolonged activation causing short and stiff fibers [6]. Compared with muscles in typically developing (TD) children, muscles in $\mathrm{CP}$ are smaller in cross sectional area, volume and muscle belly length, while Achilles tendons may be longer [7, 8]. However, studies on passive fascicle length and pennation angles have been inconclusive $[9,10]$, with some results indicating normal fascicle lengths [11] and long sarcomeres in spastic muscles [12]. Recent studies also suggest that muscle stiffness in CP already appears at an early age [13] and that maladaptation to growth, rather than spasticity, plays a crucial role in developing contractures [14].

The medial hamstrings are frequently affected by hyper-resistance in CP. In comparison to TD muscles, spastic hamstrings have been found to have lower activation thresholds during passive stretch [15] as well as altered muscle properties, including increased muscle stiffness due to larger amounts of extracellular matrix [16]. Recently, instrumented clinical tests to assess hyperresistance in the hamstrings have been developed, with which both the biomechanical and electrophysiological responses are recorded during a passive stretch $[17,18]$. Such tests yield a vast array of information about joint resistance, joint angles, angular velocity, and muscle electromyography (EMG). However, (instrumented) clinical assessment provides limited information at the underlying muscle level. This makes it difficult to distinguish between the neural and non-neural components of hyper-resistance, based on experimental data alone.

(Neuro-)musculoskeletal modeling is a powerful tool to gain insight into the underlying pathology at a more detailed level [19]. Thus far, most modeling studies of spasticity during passive stretch have focused on the ankle joint and have applied either simplified [20, 21], or highly complex [22-25] muscle models. Other modeling approaches have attempted to directly $[26,27]$ or indirectly [28] assess the effect of stretch reflexes on gait. However, the complexity of pathological muscle behavior during voluntary activation makes it very challenging to validate such models with measured data. A detailed analysis of spastic hamstring behavior during passive instrumented clinical assessment using neuro-musculoskeletal modeling could yield valuable information on tissue as well as reflective muscle behavior, but is so far lacking in literature.

Therefore, the aim of this paper was to simulate instrumented, clinical spasticity assessment of the hamstring muscles in CP using a conceptual model of contracture and spasticity. Specifically, we investigated whether we could explain 1) increased resistance during slow passive stretch (i.e. contracture) by altered passive muscle stiffness; and 2) additional increased resistance during fast passive stretch (i.e. spasticity) by a purely velocitydependent stretch reflex.

\section{Methods}

Eleven children with spastic CP (6 male, age $11.5 \pm 3.4 y$; weight $33.7 \pm 12.8 \mathrm{~kg}$ ) and 9 TD children (5 male; age $11.0 \pm 3.2 \mathrm{y} ; 34.8 \pm 13.5 \mathrm{~kg}$ ) were included in the study. Of the CP subjects, 8 were bilaterally affected and 3 unilaterally; 8 were classified as Gross Motor Function Classification System (GMFCS) level I and 3 as II. All data were collected as part of a larger study [29]. Patients were selected from the available data set if the Modified Ashworth Scale score of the hamstring muscles was 1 or higher [30], i.e. a clinical sense of mild hyper resistance ( 1 subject had a score of $1 ; 6$ of $1+$, and 4 of 2 ; with an average Modified Tardieu Score of $\left.-78.5 \pm 6.7^{\circ}\right)$. Only data on left legs were included for practical reasons. To exclude patients with high background activation or who were not relaxed, measurements with a root mean square EMG (RMS-EMG) activity during slow passive stretch higher than $10 \%$ of that measured during maximum voluntary contraction (about $2 \%$ of the stretches) were excluded. Available data of 9 TD children were used for comparison [17]. All subjects older than 11 years and all parents signed an informed consent form. The data collection protocol was approved by the medical ethics committee of the KU Leuven University Hospital.

Instrumented spasticity assessment of the left leg hamstrings was performed on all subjects (Fig. 1a; [17]). The knee was stretched at a slow ( $>5 \mathrm{~s}$ for the entire range of motion) and a fast $(<1 \mathrm{~s})$ stretch velocity, while the subject was laying supine with the left hip in $90^{\circ}$, and the pelvis and thigh fixed by a second examiner. Knee angular displacement and velocity were recorded with inertial 

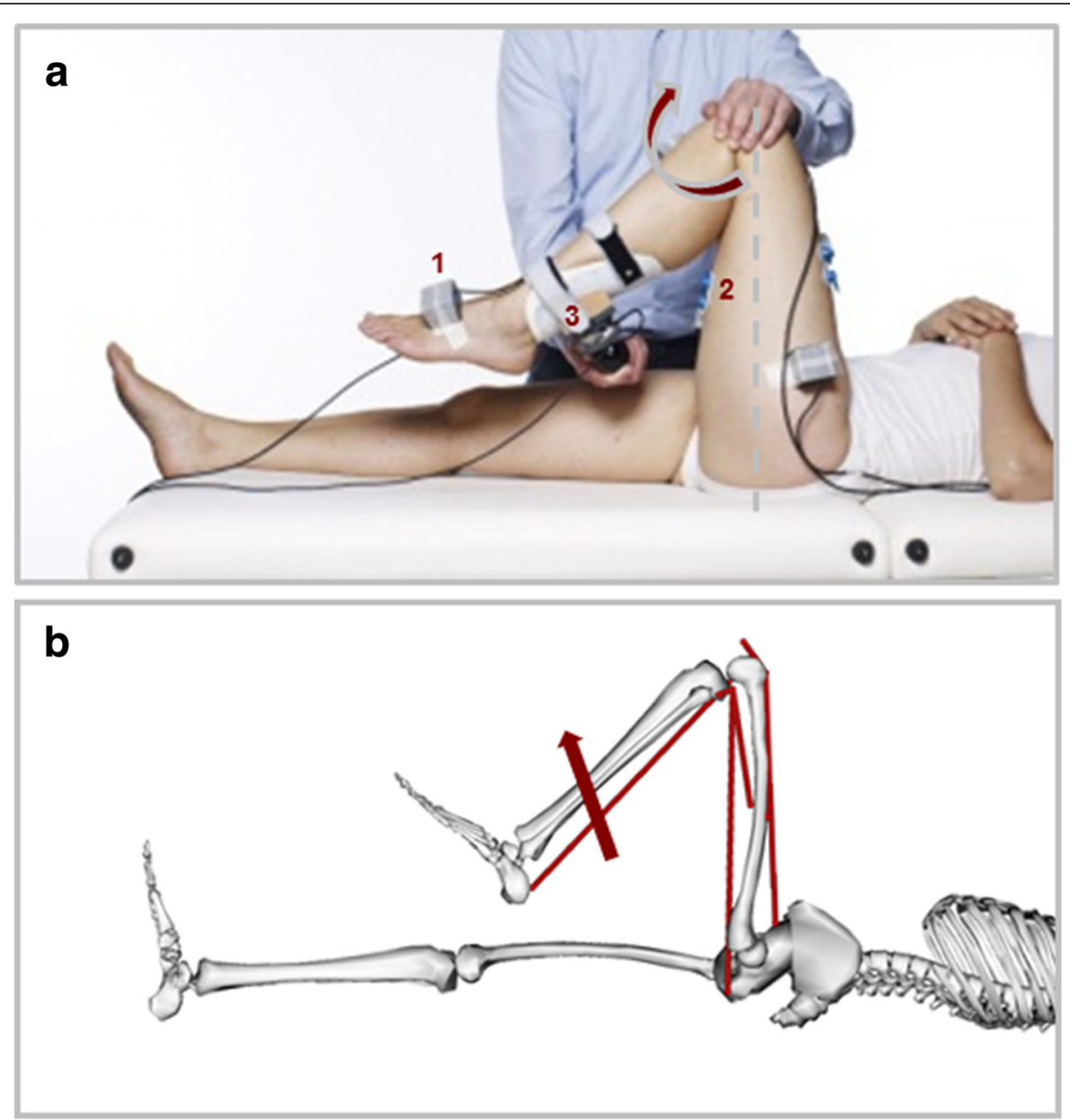

Fig. 1 Measurement and model set-up. a Measurement set-up showing (1) inertial sensors, (2) EMG electrodes and (3) hand-held 6 - DOF force transducer; $\mathbf{b}$ OpenSim model used with 5 lumped muscles around the knee, and the applied force as measured experimentally

measurement units. The force applied to the shank by the examiner was recorded with a hand-held 6 degrees of freedom (DOF) force/torque sensor. The location of force application relative to the knee joint axis was measured manually parallel and perpendicular to the tibia. Surface EMG was collected from the medial hamstrings and rectus femoris (see [17] for measurement details).

A dedicated musculoskeletal model (Fig. 1b) was developed using OpenSim software [31]. The model was adapted from the generic gait model (GAIT2392) and included torso, pelvis, and left and right femur, tibia and foot (of which torso and right leg for visualization only). All joints were locked in $0^{\circ}$, except for the left hip, which was locked in $90^{\circ}$ as imposed during all measurements, and the evaluated left knee, which was free to move until full extension. All muscles were removed except for those around the left knee, which were lumped to 5 major muscle groups: hamstrings (HAM, representing semitendinosus, semimembranosus and biceps femoris long head), vasti (VAS, representing vastus lateralis, medialis and intermedius), rectus femoris (RF), biceps femoris short head (BFS), and gastrocnemius (GAS, representing gastrocnemius lateralis and medialis). The path of the lumped muscles and the total muscle tendon length (optimal fiber length plus tendon slack length) were equal to that of one representative original muscle (biceps femoris long head for HAM, vastus intermedius for VAS, and gastrocnemius lateralis for GAS). Paths were selected based on other simplified models (e.g. Gait10dof18musc.osim) and previous literature [32]. To obtain myotendinous behavior representative of the whole muscle group, the fiber to tendon length ratio was averaged over all three muscles. The optimal muscle force was taken as the sum of the represented muscles' forces. The 'default activation' was set to 0.01 for all muscles.

The model was scaled to individual subject sizes based on the subject's height, leg length and tibia length, as measured during clinical examination. Muscle strength was scaled with the subject's mass to the $2 / 3$-power. Experimentally measured knee angles and applied forces 
were imported to OpenSim. The measured 6-DOF force was applied to the shank segment at the same location relative to the knee as measured experimentally. An inverse dynamic (ID) analysis was run in OpenSim to obtain the net knee moment, representing the sum of all (passive plus active) internal muscle moments around the knee.

As a model for contracture, passive muscle properties for HAM and VAS were optimized to match the slowvelocity net knee moment versus knee angle curve. The other three muscles were found to have negligible effect, as they were not in their end range of motion in any part of the assessment. The two parameters that determine the passive force-length curve in the Thelen muscle model [33] were adjusted, i.e. the parameters FmaxMuscleStrain ('S', the passive muscle strain at maximum isometric muscle force) and KshapePassive ('K', the exponential shape factor for the passive force-length relationship). An fminsearch optimization algorithm was used (Matlab 2010, The Mathworks), with the default values in Open$\operatorname{Sim}(\mathrm{S}=0.6 ; \mathrm{K}=4)$ as starting values, and no boundaries. To test the robustness of the optimization, additional different starting values were used, and this was found to have negligible effect. A muscle analysis was performed for each set of $\mathrm{S}$ and $\mathrm{K}$, to obtain the total muscle knee moments generated by all five muscles. The sum of all muscle moments was compared with the net knee moment from the ID analysis. In the optimization, the RMS error between the two curves was minimized. The optimal $\mathrm{S}$ and $\mathrm{K}$ values for HAM and VAS, as well as the associated RMS error were obtained for each subject.

To test how well the model could predict measured motion, forward dynamic (FD) simulations were performed for all assessments. The measured initial position and applied forces were used as input, while predicted knee angle and muscle activity were output of the simulations. The passive muscle properties $\mathrm{S}$ and $\mathrm{K}$ were set to the optimal values for each individual for all FD simulations. First, the slow stretch data were simulated for both TD and CP, to verify that the model was capable of replicating data using FD simulations. Second, the fast stretch data were simulated to predict the effect of stretch reflexes in CP, and to obtain a reference in TD.

To model spasticity during the CP fast stretch simulations, a stretch reflex controller was developed in OpenSim. This controller imposed a velocity-dependent muscle excitation any time a fiber was stretched above a certain threshold velocity, according to:

$$
E_{m}\left(t+t_{d}\right)=\left\{\begin{array}{r|c}
G \cdot v_{m}(t) & v_{m}(t)>T \\
0 & v_{m}(t) \leq T
\end{array}\right.
$$

with $E_{m}$ the muscle excitation for muscle $m, t_{d}$ a time delay factor representing the stretch reflex delay, $G$ a gain factor representing the severity of the enhanced reflex, $v_{m}$ the fiber velocity of muscle $\mathrm{m}$, and $T$ the threshold velocity. The controller was implemented as a plug-in in OpenSim and is freely available on https://simtk.org/ home/spasticitymodel [34]. $t_{d}$ was set to a fixed value of $30 \mathrm{~ms}$, representing the shortest possible stretch reflex delay as found in the literature [35]. Based on the measured data, threshold values were determined as the stretch velocity $30 \mathrm{~ms}$ before onset of EMG of the muscle-tendon complex (thus assuming that muscle fiber velocity equals muscle-tendon velocity, which is reasonable considering the low forces). EMG onset was identified using the method of Staude and Wolf [36]. Gain values were set to 0 , 1, 2 and 4 for each subject, which was found to cover the range of potential values. As no notable EMG activity was seen in any of the TD subjects' fast stretches, the stretch reflex controller was only applied during $\mathrm{CP}$ fast stretch simulations.

To validate the FD results, predicted knee angles were compared with measured knee motion, and muscle excitation as evoked by the stretch reflex controller was compared with measured EMG. Both comparisons were done qualitatively by looking at the graphs, and for knee motion also quantitatively by calculating the RMS difference between the curves. A distinction was made between the stretch phase, in which the knee was moved quickly to its end range of motion, and the hold phase, in which the knee was held stationary at this end range of motion. In total $1 \mathrm{~s}$ of data was simulated for all FD simulations. The muscle fiber length and velocity during the $\mathrm{CP}$ fast stretch simulations were also analyzed, to gain further insight in the working mechanisms of the stretch reflex controller.

For statistical analysis, the optimal S and K parameters and the RMS error of the optimization were compared between TD and CP using a Mann-Whitney U test. This non-parametric test was chosen because of the small sample and non-normally distributed data. The RMS errors of the FD simulations for knee angle were also compared between TD and CP using a Mann-Whitney U test, and between different gain values in $\mathrm{CP}$ using a KruskalWallis test. The level of significance was set to $p<0.05$.

\section{Results}

\section{Optimization of passive muscle properties}

The net knee moments as obtained from the ID analysis revealed that the $\mathrm{CP}$ children had a steeper knee momentangle curve (Fig. 2a, dashed lines), and hence overall stiffer knee extensor muscles (vasti and rectus femoris) and knee flexor muscles (hamstrings and gastrocnemius). These measured net knee moment-angle curves could almost perfectly be replicated by optimizing $\mathrm{K}$ and $\mathrm{S}$ for hamstrings and vasti (Fig. 2a, solid lines). The fit for $\mathrm{CP}$ was slightly better than for TD, with an RMS error between the 

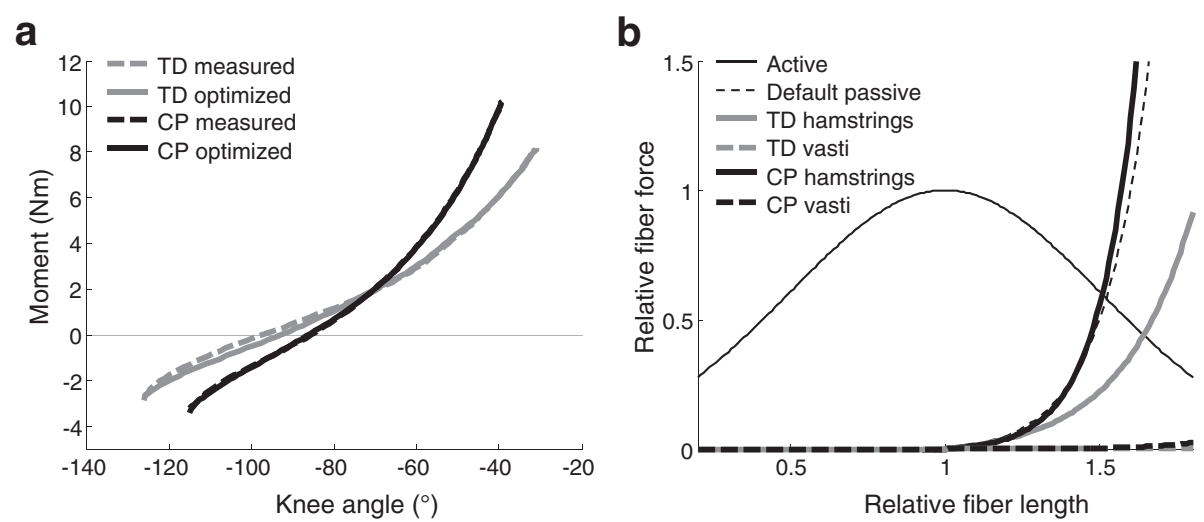

Fig. 2 Knee moment-angle and muscle force-length curves. a Knee moment-angle curve for typically developing (TD, grey) and cerebral palsy $(\mathrm{CP}$, black) children, averaged over all subjects. Inverse dynamic results of the measurement are shown (dashed), as well as the optimized results of the muscle analysis (solid). b. Active and passive muscle force-length curves, based on the optimized passive properties $\mathrm{S}$ and $\mathrm{K}$ as averaged over all TD (thick grey) and CP (thick black) subjects, for hamstrings (solid) and vasti (dashed). The TD vasti curve mostly underlies the CP vasti curve. Default active and passive curves (thin lines) are based on Thelen (2003) and typically assumed equal for all muscles, both only scaling in amplitude to maximum isometric muscle force [33]

measured and modeled knee moment of $0.10 \pm 0.06 \mathrm{Nm}$ for CP and $0.24 \pm 0.12 \mathrm{Nm}$ for TD $(p=0.004$, Table 1$)$.

Figure $2 \mathrm{~b}$ shows the passive force-length curves for these optimized $\mathrm{S}$ and $\mathrm{K}$ values, averaged over all TD and $\mathrm{CP}$ subjects. The $\mathrm{CP}$ hamstrings had significantly lower S values compared with TD, with only $57 \pm 11 \%$ stretch at optimal muscle force versus $82 \pm 20 \%$ for TD $(p=0.004$; Table 1). Shape factor K tended to be higher in $\mathrm{CP}(p=0.057)$, indicating a more concave curve in $\mathrm{CP}$ than in TD. For the vasti, $\mathrm{S}$ and $\mathrm{K}$ both tended to be lower in $\mathrm{CP}$, but these differences were not significant $(p=0.08$ and $p=0.32$ respectively). All muscles were

Table 1 Optimization and forward dynamic simulation results

\begin{tabular}{|c|c|c|c|c|c|}
\hline & & $\mathrm{TD}(N=9)$ & $\mathrm{CP}(N=11)$ & Effect size & $p$-value \\
\hline & & mean \pm std & mean \pm std & & \\
\hline Muscle properties & & & & & \\
\hline & S hamstrings & $0.77 \pm 0.15$ & $0.57 \pm 0.11$ & -0.20 & 0.008 \\
\hline & $\mathrm{K}$ hamstrings & $3.59 \pm 0.63$ & $4.65 \pm 1.27$ & 1.07 & 0.057 \\
\hline & S vasti & $4.21 \pm 8.19$ & $1.40 \pm 1.73$ & -2.82 & 0.080 \\
\hline & K vasti & $16.12 \pm 15.28$ & $8.73 \pm 3.95$ & -7.40 & 0.322 \\
\hline & optimization error (Nm) & $0.24 \pm 0.12$ & $0.10 \pm 0.06$ & -0.14 & 0.004 \\
\hline FD RMS errors $\left(^{\circ}\right)$ & & & & & \\
\hline & FD slow & $3.58 \pm 2.38$ & $1.12 \pm 0.95$ & -2.46 & 0.006 \\
\hline Stretch Only & FD fast - no spas & $6.17 \pm 3.60$ & $19.20 \pm 6.76$ & 13.03 & 0.000 \\
\hline & FD fast - spas G1 & & $14.59 \pm 7.28$ & & \\
\hline & FD fast - spas G2 & & $9,00 \pm 5,08$ & & \\
\hline & FD fast - spas G4 & & $9.61 \pm 5.94$ & & \\
\hline & FD fast - spas $G_{o p t}$ & & $5.95 \pm 2.13$ & & $0.766^{\mathrm{a}}$ \\
\hline Stretch + Hold & FD fast - no spas & $10.55 \pm 5.62$ & $18.12 \pm 5.81$ & 7.57 & 0.016 \\
\hline & FD fast - spas G1 & & $17.85 \pm 9.25$ & & \\
\hline & FD fast - spas G2 & & $15.27 \pm 8.60$ & & \\
\hline & FD fast - spas G4 & & $12.19 \pm 4.75$ & & \\
\hline & FD fast - spas $G_{o p t}$ & & $10.40 \pm 4.59$ & & $0.941^{\mathrm{a}}$ \\
\hline
\end{tabular}

Abbreviations: $T D$ typically developing, $C P$ cerebral palsy, $S$ passive muscle strain at optimum muscle force, $K$ shape factor of passive force-length curve, $F D$ forward dynamic results, G1-4 stretch reflex gain factors of 1 to $4, G_{\text {opt }}$ optimal gain factor $(0,1,2$, or 4$)$ per subject

${ }^{a} S p a s G_{o p t}$ results were statistically compared against TD no spas 
found to be much more compliant than the default OpenSim Thelen muscle, except for the CP hamstring muscles.

\section{Forward dynamic results}

The knee angle curve during slow passive stretch was almost perfectly replicated in the FD simulations for all $\mathrm{CP}$ children, with an RMS error of $1.1 \pm 0.9^{\circ}$ (Fig. $3 \mathrm{~b}$ ), thus indicating that predictive FD simulations of the clinical tests were accurate when the correct parameters were chosen. For the TD children, the fits were slightly less good, but still reasonable (RMS error of $3.6 \pm 2.4^{\circ}$, Fig. 3a).

Even though passive muscle stiffness was not optimized for the fast stretches, the FD simulated knee angles for TD matched well with the measured knee angles, except for on average a small overshoot towards the end range of motion (Fig. 3c). The RMS error for the stretch phase only (until $0.6 \mathrm{~s}$ in TD) was $6.2^{\circ} \pm 3.6^{\circ}$ (Table 1). For all but one CP subjects, the FD simulations without spasticity were clearly overshooting the measured knee angle (Fig. 3d). Adding spasticity reduced the overshoot and improved the fit considerably. Table 2 shows the RMS error values of predicted versus measured knee angles during fast stretches in children with $\mathrm{CP}$ obtained with different gain values in the stretchreflex controller. The optimal gain, i.e. the gain with the lowest RMS error during the stretch phase (until $0.4 \mathrm{~s}$ in CP), is indicated per subject in bold. Most subjects had a best fit with a gain of 2 or 4, while two had a lower score with a gain of 1 and one had a best fit with a gain of zero (i.e. no spasticity). The average RMS error for predicting the knee angle with the most optimal gain value was of $5.9 \pm 2.1^{\circ}$, which was comparable to the value for the TD children without the addition of spasticity. Towards the end range of motion, almost all subjects still had an overshoot even with the inclusion of spasticity (Fig. 3d), as also illustrated by an approximately $4^{\circ}$ higher RMS error for the complete stretch and hold phase, compared with only the stretch phase (Table 1).

\section{Muscle fiber activity, length and velocity}

Muscle activity came on (by definition) $30 \mathrm{~ms}$ after the stretch reflex controller was activated, and for all subjects the measured and simulated muscle activity was qualitatively very comparable during the main part of the stretch phase (several examples in Fig. 4). However, after the stretch phase, sustained EMG was seen in the experimental data, which was not predicted by our purely velocitydependent spasticity model.

The sudden increase in muscle activation as illustrated in Fig. 4 resulted in a clear break in the muscle fiber length and velocity and thus in a lower peak fiber length and peak fiber velocity, as illustrated for a typical
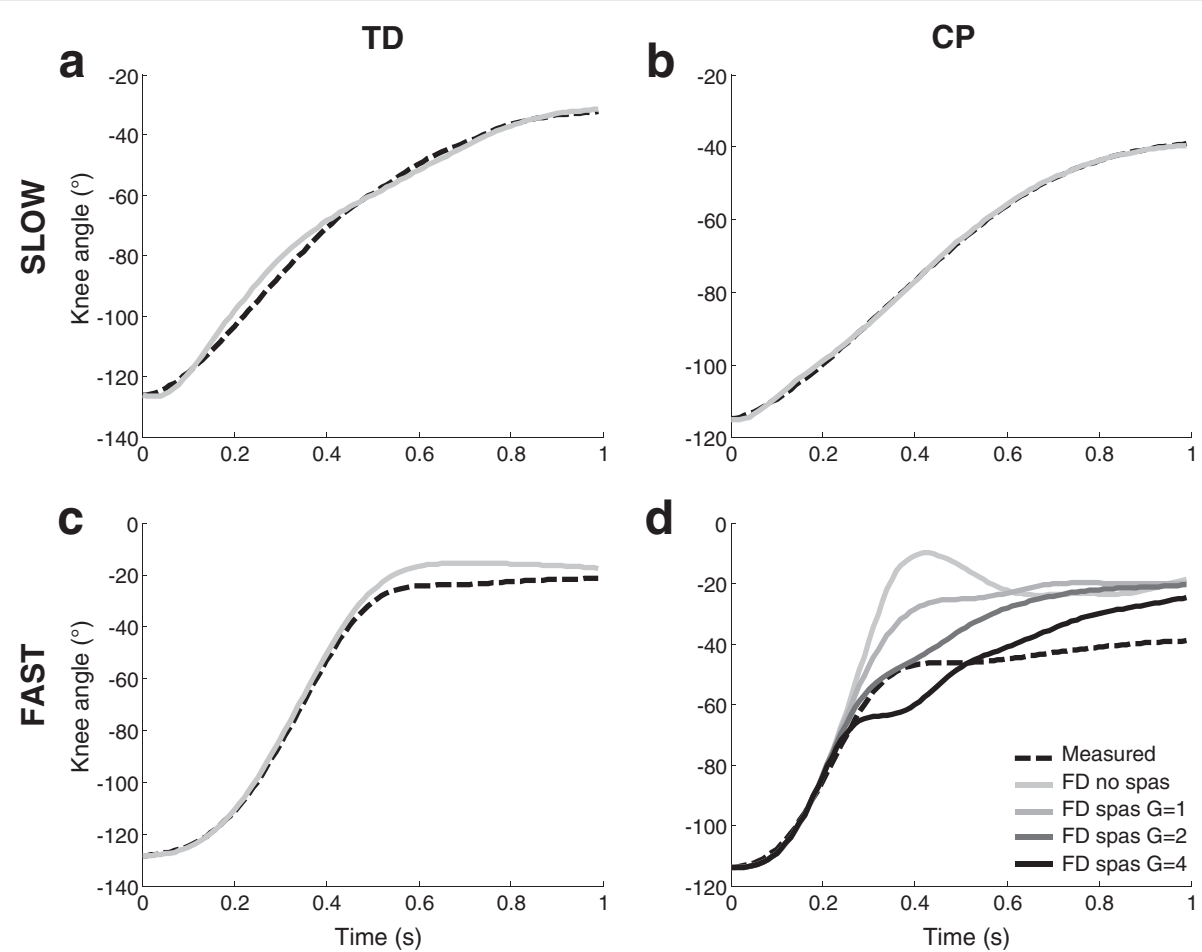

Fig. 3 Forward-dynamic (FD) predicted (solid lines) versus measured (dashed lines) knee angles over time, for a typically developing (TD) slow; b cerebral palsy (CP) slow; c TD fast and $\mathbf{d}$ CP fast. Data are averaged over all subjects. For CP fast stretches, results including the stretch reflex controller with increasing gain $(G)$ values are shown 
Table 2 Individual RMS errors $\left(^{\circ}\right)$ of predicted versus measured knee angles during the stretch phase

\begin{tabular}{lcccc}
\hline Subject & Gain $=0$ & Gain $=1$ & Gain $=2$ & Gain $=4$ \\
\hline CP01 & 29.60 & 13.99 & $\mathbf{5 . 1 3}$ & 5.45 \\
CP02 & 14.45 & 10.13 & 5.77 & $\mathbf{4 . 8 5}$ \\
CP03 & 22.95 & 14.64 & $\mathbf{7 . 5 1}$ & 7.89 \\
CP04 & 24.49 & 23.48 & 12.29 & $\mathbf{9 . 0 6}$ \\
CP05 & $\mathbf{8 . 3 4}$ & 13.51 & 18.93 & 25.30 \\
CP06 & 26.12 & 18.98 & 13.33 & $\mathbf{7 . 4 1}$ \\
CP07 & 12.03 & $\mathbf{6 . 3 2}$ & 7.30 & 14.60 \\
CP08 & 19.47 & 26.96 & 12.77 & $\mathbf{6 . 4 1}$ \\
CP09 & 12.05 & 4.19 & $\mathbf{2 . 6 9}$ & 8.28 \\
CP10 & 18.61 & 7.77 & $\mathbf{2 . 4 9}$ & 11.27 \\
CP11 & 23.08 & 20.46 & 10.78 & $\mathbf{5 . 2 3}$ \\
\hline
\end{tabular}

Lowest RMS-error values per subject are indicated in bold

example $\mathrm{CP}$ subject in Fig. 5. With increasing gain values, more spiky patterns in length, velocity and activity were found.

\section{Discussion}

In this study, an individually tunable, conceptual model of contracture and spasticity was developed, and tested on instrumented clinical assessment of the hamstrings in children with $\mathrm{CP}$ and TD children. It was found that increased resistance during slow passive stretch in both TD and CP could be simulated well by only adjusting passive muscle stiffness. Fast passive stretch could be reasonably well replicated for TD, while the addition of a purely velocity-dependent stretch reflex controller significantly improved the fast stretch simulations in CP.

Muscle stiffness parameters $\mathrm{S}$ and $\mathrm{K}$ were highly variable between subjects and substantially different in both TD children and children with CP from the generic muscle model parameters derived from Thelen [33]. Particularly for the vasti, we found a gross underestimation of the original values indicating excessive passive stiffness in the generic model. This is in line with previous studies also showing excessive passive stiffness of the quadriceps for this same model [37, 38], and the present results emphasize the magnitude of this discrepancy. In the gastrocnemius, the opposite has been reported, with the generic model indicating less passive stiffness than experimentally determined for both TD and CP [39]. These findings affirm the importance of individually tuning model parameters that aim to explain healthy and pathological pediatric muscle properties.

In line with previous findings [40], we did not find significant differences between $\mathrm{CP}$ and TD children for the passive stiffness properties of the vasti. This may be due
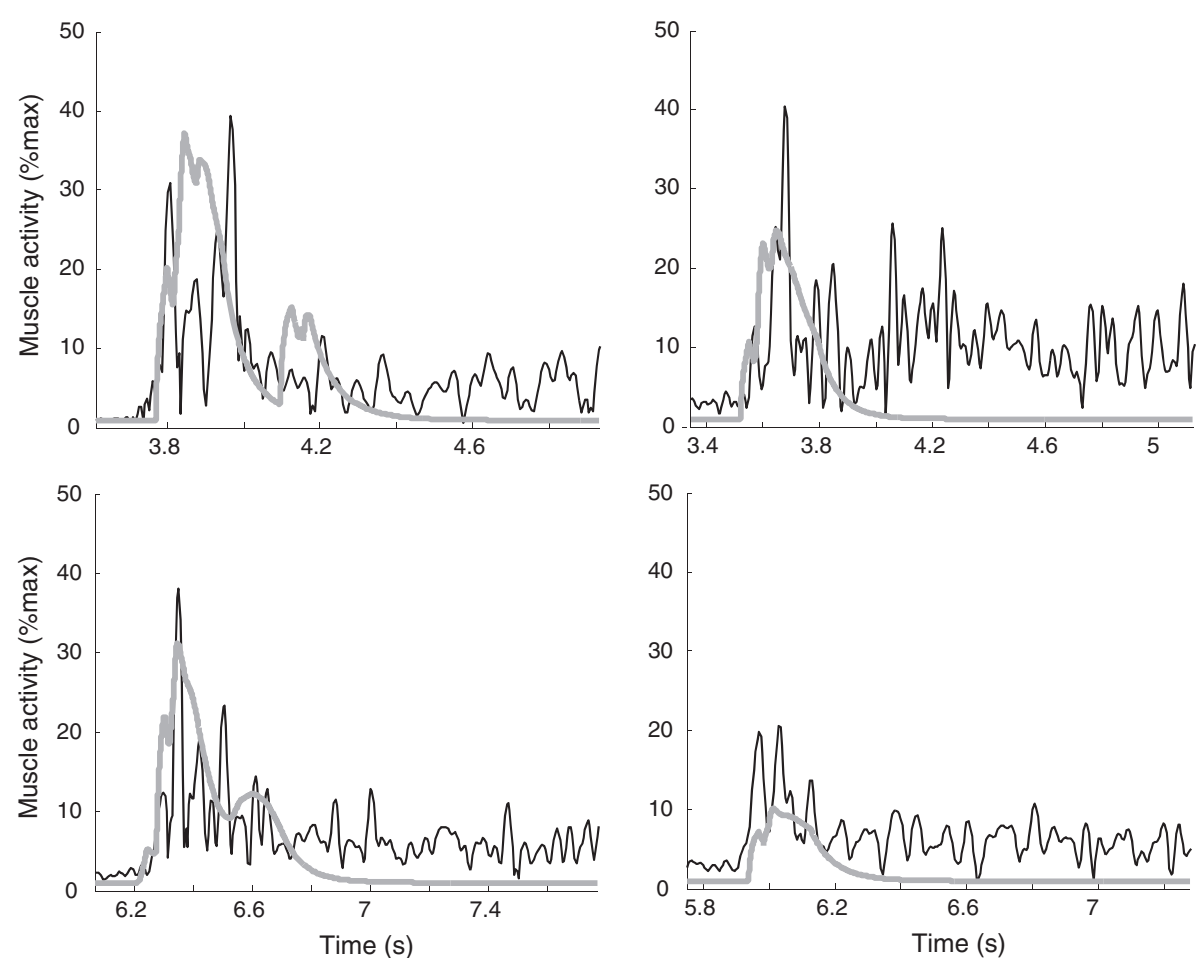

Fig. 4 Measured EMG (thin black lines) and simulated (thick grey lines) muscle activity. Data are shown for four typical example CP children. Both EMG and simulated muscle activity are shown as a percentage of maximum, for the measured data this maximum was determined during maximum isometric contraction. For each subject, the simulation with the individually tuned optimal gain value is shown 


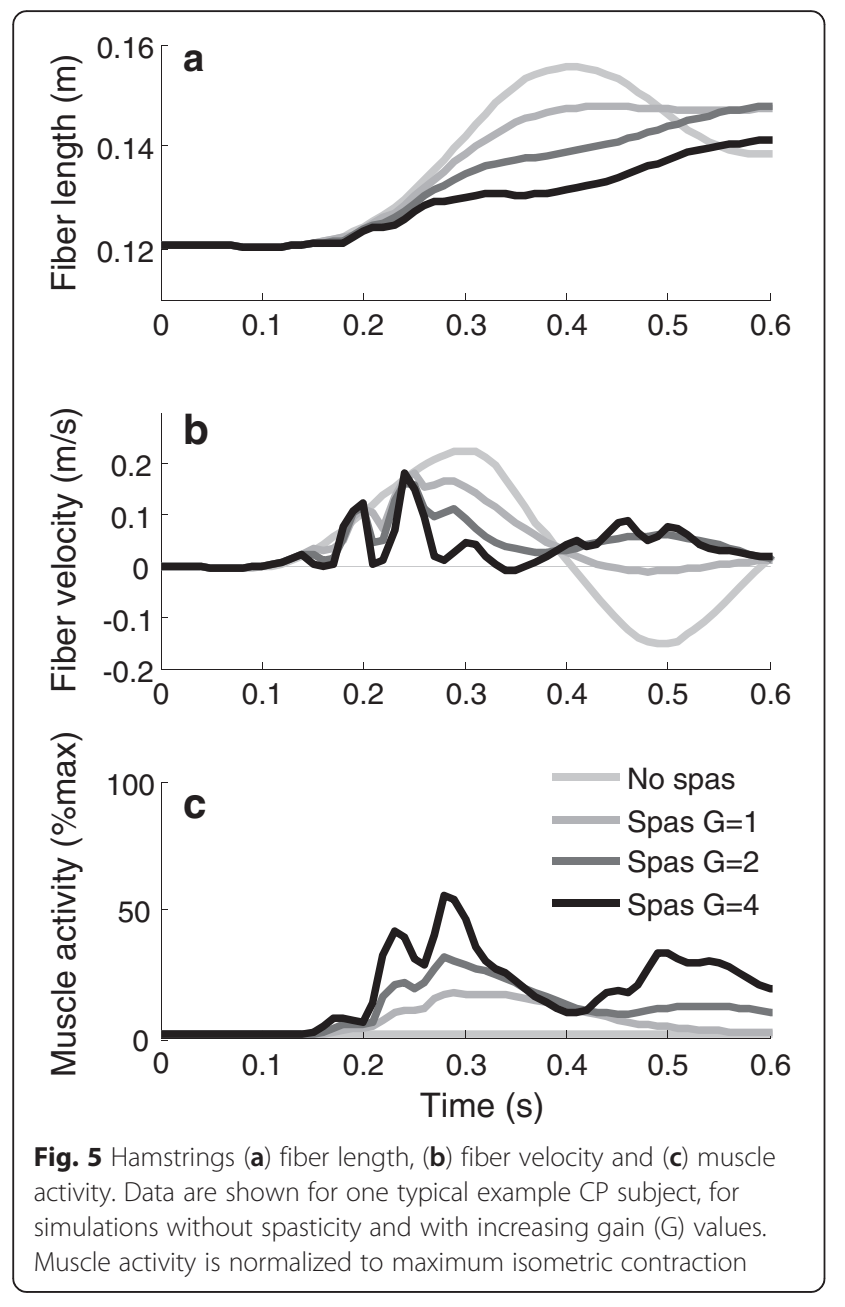

to the large spread in the data and the difficulty of accurately estimating these parameters separately, especially for TD. This was likely related to the fact that the vasti muscles were not fully stretched to their end range of motion in our experimental data. For this reason, $\mathrm{S}$ and $\mathrm{K}$ were more dependent on each other for the vasti than for the hamstrings, as the stiffness curve was only in its lag phase and not shaped enough to give a good estimate for both $\mathrm{S}$ and $\mathrm{K}$. As our focus was on the hamstring muscles, the vasti stiffness parameters were mainly used to allow for good predictive simulations. To better estimate the stiffness parameters for the quadriceps, measurements up to full knee flexion should be included.

Unlike previous modeling studies [39, 41], we opted to alter the passive properties of the hamstrings and vasti muscle tissue rather than altering its gross morphology. Specifically for the medial hamstrings, Smith et al. [16] has shown that increased collagen content in the muscles' extra cellular matrix is a main contributor to increased muscle stiffness. Furthermore, examination of biopsies taken from the flexor carpi ulnaris of children with $\mathrm{CP}$ and TD children indicated significant increases in the connective tissue reinforcement of neurovascular tissues penetrating the muscle [42]. Yet, several studies have also reported that muscles from subjects with $\mathrm{CP}$ have reduced muscle volumes, shorter muscle bellies and shorter optimal fiber lengths compared to those of TD children [7, 16, 43]. Our finding that altered passive muscle stiffness could fully explain the experimentally measured moment angle curves during slow stretch, indicates that our contracture model may capture all these underlying causes of passive muscle stiffness, without changing the active muscle behavior.

During fast passive stretch, altering the passive muscle properties alone resulted in a reasonable simulation in TD, but not in CP. The small overshoot seen in TD (Fig. 3c) may be due to viscosity, which was not included in our model. Reflex activity is not likely to play a role in TD fast stretches, as no notable EMG activity was seen in any of the TD subjects, and hence this was not included in our simulations. In CP, a much larger overshoot was seen during fast passive stretches, and adding velocity-dependent reflex activation to the hamstrings significantly improved the fit to the level of TD. This demonstrates that the purely velocity-dependent controller could predict much of the increased resistance due to fast stretch. The large variation in gain factors among the subjects (Table 2) indicates the variability in spastic reactions among children with CP. For example, one subject with $\mathrm{CP}$ had hardly any overshoot in his kinematics during a fast stretch, no difference between measured and simulated end range of motion, and a best fit with measured data if no spasticity was included (Table 2, CP05). Interestingly, this was also the subject with the lowest measured RMS EMG during the fast stretch and also the lowest clinical Modified Ashworth Scale (1, where all others were $1+$ or higher). Thus, spasticity played only a minor role in this subject, which was confirmed by our simulations. Current clinical assessment scores, such as the Modified Ashworth, tend to cluster muscles into broad severity categories, thereby limiting their ability to differentiate between subjects or to detect response to treatment. Conversely, the proposed model allows data from the instrumented assessment to be individually tuned, therefore providing a wider range of possible outcomes with which to compare subjects or study the effect of treatment.

Prolonged muscle activation after the fast passive stretch in CP was not predicted by our model and may, together with viscosity, help explain some discrepancies between the measured and simulated kinematics during the end range of motion in CP. Such activation may be contradictory to the classical definition of spasticity as being velocity-dependent [4], but is in line with multiple experimental studies that have reported tonic activation during passive stretch of spastic muscles [44-47], and particularly in the hamstrings [15]. A possible explanation for 
the continued activation may be that alterations in the membrane properties of alpha motor neurons increase their sensitivity to afferent input. This in turn triggers persistent inward currents (PIC) that lead to prolonged depolarization states called plateau potentials. Following loss of normal central regulation, PIC and plateaus can result in continuous low-level motor output [48]. Alternatively, length-dependent activity, even at slow stretch, has been found during passive muscle stretch especially in the hamstrings [15], and may help explain the prolonged activity. However, the amplitude of length-dependent activity during slow stretch was low in subjects selected for the current study, so this is likely only a minor effect.

Although these different mechanisms may all play a role, we believe that model complexity should be, in first instance, avoided. A model with many parameters may fit well with the measured data (low bias), but the accuracy with which each parameter can be estimated is prone to being compromised (high variance). Additionally, when multiple dependent parameters are included in a model, optimization algorithms tend to adjust only that parameter that has the most influence on the error, even though experimental data may show otherwise. In this study, we attempted to model the measured data with as low a number of optimizable model parameters as possible, choosing those parameters that are physiologically most likely to be altered in CP. As length, velocity, and force (or acceleration) feedback are highly interdependent, distinguishing between their effects, based on externally measured data or simulations, will be difficult. Jansen et al. [26] included both length and velocity feedback in forward-dynamic simulations of gait, and found that both feedback types resulted in pathological gait characteristics as seen post stroke. The effect of length and velocity feedback were however highly similar and therefore difficult to distinguish. Similarly, the effects of increased muscle stiffness, increased tendon stiffness, and reduced fiber and tendon length are highly related and would all result in steeper joint moment-angle curves if included in the model. We chose not to alter tendon length or stiffness, as there is limited evidence that these contribute to the overall hamstrings stiffness in $\mathrm{CP}$, while Achilles tendons in $\mathrm{CP}$ have even been found to be more compliant [49-51]. Finally, we also had to lump the hamstrings and vasti muscle groups, as many of the individual muscle parameters were interdependent, and hence it was not possible to reliably estimate them separately. The resulting lumped hamstring is not representative of any of the individual hamstrings heads on its own, but rather of the total muscle, allowing us to better understand the overall behavior. The absolute values of the model parameters are likely influenced by the path chosen and would have been slightly different had we chosen for instance the semimembranosus or semitendinosus path. However, the differences between $\mathrm{CP}$ and TD are not expected to be influenced by this effect, and our conclusions are therefore independent of the muscle path chosen.

In future studies, richer experimental data sets, for instance including active force generating tests and ultrasound measurements, could provide additional information about the underlying muscle properties and behavior. This could allow for more accurate estimation of additional model parameters such as fiber and tendon lengths and active muscle properties, as well as a better distinction between the individual hamstrings heads. Simulation of additional data sets per muscle, application to other joints and muscles, and inclusion of a larger sample of subjects could also help to further validate our conceptual model for contracture and spasticity. If complemented with personalized skeletal geometry, for instance as obtained from MR images [52, 53], comprehensive patient-specific models could be generated.

As such, our individually tuned model for contracture and spasticity could be applied to simulate more clinically relevant tasks such as gait. In this way, the model may help identify to what extent passively measured (pathological) muscle properties and motor control affect dynamic tasks, and should be subject to treatment. To help speed up such applications, the stretch reflex controller as well as the underlying source code are made freely available online on the simtk website [34].

\section{Conclusion}

This paper shows that passive muscle properties, i.e. muscle strain $\mathrm{S}$ and shape factor $\mathrm{K}$, of hamstrings and vasti can be individually estimated based on slow stretch instrumented spasticity assessment data, and can fully explain muscle behavior during slow passive stretch. Hamstring muscle properties were found to be stiffer in $\mathrm{CP}$ and variable between subjects. A purely velocity-dependent spasticity model (individually tuned for its Gain) can predict muscle response during fast passive stretch in terms of predicted knee angle, muscle activity, fiber length and velocity. Only after the fast stretch phase, simulated and measured knee angles and muscle activity started to deviate, as sustained muscle activity, not dependent on velocity, was not predicted by our model.

\section{Additional file}

Additional file 1: Individual subject data. (XLSX $22 \mathrm{~kb}$ )

\section{Abbreviations}

BFS, Biceps femoris short head; CP, Cerebral palsy; DOF, degrees of freedom; EMG, Electromyography; FD, Forward dynamics; GAS,

Gastrocnemius lateralis and medialis; GMFCS, Gross Motor Function Classification System; HAM, Hamstrings muscle group; ID, Inverse dynamics; K, KshapePassive, exponential shape factor for the passive force-length 
relationship; PIC, persistent inward currents; RF, Rectus femoris; RMS, Root mean square; $\mathrm{S}$, FmaxMuscleStrain, passive muscle strain at maximum isometric muscle force; TD, Typically developing; VAS, Vasti muscle group

\section{Acknowledgements}

We greatly acknowledge the OpenSim team, in particular Ajay Seth and Ayman Habib, for their help in developing the stretch reflex controller plug-in. We also thank Ilse Jonkers and Friedl de Groote for assistance with our optimizations, and Lizeth Sloot for detailed proof reading of the manuscript.

\section{Funding}

This work was funded by NCSRR Visiting Scholar and Pilot Project Program grants to MK, supported by NIH research infrastructure grant R24 HD065690. LB was supported by PhD grant DBOF/12/058 and FWO post-doc grant 12R4215N. None of these funding sources had any role in the design of the study, the collection, analysis, and interpretation of data, nor in writing the manuscript

\section{Availability of data and materials}

The individual subject data for Table 1 on which the conclusions are based are available as Additional file 1 to this paper.

\section{Authors' contributions}

MK implemented the OpenSim models and developed the stretch reflex controller and the simulations. LB performed the data collection and analysis, executed simulations, and performed the statistical analyses. MK and LB were both responsible for the data interpretation and writing of the manuscript. TK assisted in the development of the simulations and performed a large set of pilot simulations. $\mathrm{KD}$ and JH assisted in the design of the study and helped to draft the manuscript. All authors read and approved the final manuscript.

\section{Competing interests}

The authors declare that they have no competing interests.

\section{Consent for publication}

Not applicable.

\section{Ethics approval and consent to participate}

The data collection protocol was approved by the medical ethics committee of the KU Leuven University Hospital (\#B32220072814). All subjects older than 11 years and all parents signed an informed consent form.

\section{Author details}

'Department of Rehabilitation Medicine, VU University Medical Center, MOVE Research Institute Amsterdam, PO Box 7057, 1007 MB Amsterdam, The Netherlands. ${ }^{2}$ Department of Rehabilitation Sciences, KU Leuven, Tervuursevest 101, B-3001 Leuven, Heverlee, Belgium. ${ }^{3}$ Clinical Motion Analysis Laboratory, University Hospital Leuven, Weligerveld 1, 3212 Pellenberg, Belgium

\section{Received: 3 November 2015 Accepted: 29 June 2016}

\section{Published online: 16 July 2016}

\section{References}

1. Rosenbaum P, Paneth N, Leviton A, Goldstein M, Bax M, Damiano D, Dan B, Jacobsson B. A report: the definition and classification of cerebral palsy April 2006. Dev Med Child Neurol Suppl. 2007;109:8-14.

2. Himmelmann K, Hagberg G, Uvebrant P. The changing panorama of cerebral palsy in Sweden. X. Prevalence and origin in the birth-year period 1999-2002. Acta Paediatr. 2010:99:1337-43.

3. Gage JR. The Identification and Treatment of Gait Problems in Cerebral Palsy. 2nd edition. London: Mac Keith Press; 2009.

4. Lance JW. The control of muscle tone, reflexes, and movement: Robert Wartenbeg Lecture. Neurology. 1980;30:1303.

5. Alhusaini AAA, Crosbie J, Shepherd RB, Dean CM, Scheinberg A. No change in calf muscle passive stiffness after botulinum toxin injection in children with cerebral palsy. Dev Med Child Neurol. 2011:53:553-8.

6. Morrell DS, Pearson JM, Sauser DD. Progressive Bone and Joint Abnormalities of the Spine and Lower Extremities in Cerebral Palsy. Radiographics. 2002;22:257-68.

7. Fry NR, Gough M, Shortland AP. Three-dimensional realisation of muscle morphology and architecture using ultrasound. Gait Posture. 2004;20:177-82.
8. Hösl M, Böhm H, Arampatzis A, Döderlein L. Effects of ankle-foot braces on medial gastrocnemius morphometrics and gait in children with cerebral palsy. J Child Orthop. 2015;9:209-19.

9. Mathewson MA, Lieber RL. Pathophysiology of Muscle Contractures in Cerebral Palsy. Phys Med Rehabil Clin N Am. 2015;26:57-67.

10. Malaiya R, McNee AE, Fry NR, Eve LC, Gough M, Shortland AP. The morphology of the medial gastrocnemius in typically developing children and children with spastic hemiplegic cerebral palsy. J Electromyogr Kinesiol. 2007;17:657-63.

11. Shortland AP, Harris CA, Gough M, Robinson RO. Architecture of the medial gastrocnemius in children with spastic diplegia. Dev Med Child Neurol. 2002;44:158-63.

12. Lieber RL, Fridén J. Intraoperative measurement and biomechanical modeling of the flexor carpi ulnaris-to-extensor carpi radialis longus tendon transfer. J Biomech Eng. 1997;119:386-91.

13. Barber L, Hastings-Ison T, Baker R, Barrett R, Lichtwark G. Medial gastrocnemius muscle volume and fascicle length in children aged 2 to 5 years with cerebral palsy. Dev Med Child Neurol. 2011:53:543-8.

14. Švehlík M, Leistritz L, Kraus T, Zwick EB, Steinwender G, Linhart WE. The growth and the development of gastro-soleus contracture in cerebral palsy. Gait Posture. 2013;38:S12

15. Bar-On L, Aertbeliën E, Molenaers G, Desloovere K. Muscle activation patterns when passively stretching spastic lower limb muscles of children with cerebral palsy. PLoS One. 2014;9, e91759.

16. Smith LR, Lee KS, Ward SR, Chambers HG, Lieber RL. Hamstring contractures in children with spastic cerebral palsy result from a stiffer extracellular matrix and increased in vivo sarcomere length. J Physiol. 2011;589:2625-39.

17. Bar-On L, Aertbeliën E, Wambacq H, Severijns D, Lambrecht K, Dan B, Huenaerts C, Bruyninckx H, Janssens L, Van Gestel L, Jaspers E, Molenaers G, Desloovere K. A clinical measurement to quantify spasticity in children with cerebral palsy by integration of multidimensional signals. Gait Posture. 2013;38:141-7.

18. Bar-On L, Aertbeliën E, Molenaers G, Van Campenhout A, Vandendoorent B, Nieuwenhuys A, Jaspers E, Hunaerts C, Desloovere K. Instrumented assessment of the effect of Botulinum Toxin-A in the medial hamstrings in children with cerebral palsy. Gait Posture. 2014;39:17-22.

19. Delp SL, Loan JP, Hoy MG, Zajac FE, Topp EL, Rosen JM. An interactive graphics-based model of the lower extremity to study orthopaedic surgical procedures. IEEE Trans Biomed Eng. 1990:37:757-67.

20. Bar-On L, Desloovere K, Molenaers G, Harlaar J, Kindt T, Aertbeliën E. Identification of the neural component of torque during manually-applied spasticity assessments in children with cerebral palsy. Gait Posture. 2014;40:346-51.

21. Harlaar J, Becher JG, Snijders CJ, Lankhorst GJ. Passive stiffness characteristics of ankle plantarflexors in hemiplegia. Clin Biomech. 2000;15:261-70.

22. Sloot LH, van der Krogt MM, de Gooijer-van de Groep KL, van Eesbeek S, de Groot J, Buizer Al, Meskers C, Becher JG, de Vlugt E, Harlaar J. The validity and reliability of modelled neural and tissue properties of the ankle muscles in children with cerebral palsy. Gait Posture. 2015:42:7-15.

23. De Gooijer-van de Groep KL, de Vlugt E, de Groot JH, van der HeijdenMaessen HCM, Wielheesen DHM, van Wijlen-Hempel RMS, Arendzen JH, Meskers CGM. Differentiation between non-neural and neural contributors to ankle joint stiffness in cerebral palsy. J Neuroeng Rehabil. 2013;10:81

24. Willerslev-Olsen M, Lorentzen, Nielsen JB, Sinkjaer T. Passive muscle properties are altered in children with cerebral palsy before the age of 3 years and are difficult to distinguish clinically from spasticity. Dev Med Child Neurol. 2013:55:617-23.

25. De Vlugt $\mathrm{E}$, de Groot JH, Schenkeveld KE, Arendzen JH, van der Helm FCT, Meskers CGM. The relation between neuromechanical parameters and Ashworth score in stroke patients. J Neuroeng Rehabil. 2010;7:35.

26. Jansen K, De Groote F, Aerts W, De Schutter J, Duysens J, Jonkers I. Altering length and velocity feedback during a neuro-musculoskeletal simulation of normal gait contributes to hemiparetic gait characteristics. J Neuroeng Rehabil. 2014;11:78.

27. Sinkjaer T, Andersen JB, Nielsen JF, Hansen HJ. Soleus long-latency stretch reflexes during walking in healthy and spastic humans. Clin Neurophysiol. 1999;110:951-9.

28. Van der Krogt MM, Doorenbosch CAM, Becher JG, Harlaar J. Dynamic spasticity of plantar flexor muscles in cerebral palsy gait. J Rehabil Med. 2010:42:656-63.

29. Bar-On L, Molenaers G, Aertbeliën E, Monari D, Feys H, Desloovere K. The relation between spasticity and muscle behavior during the swing phase of gait in children with cerebral palsy. Res Dev Disabil. 2014;35:3354-64. 
30. Bohannon RW, Smith MB. Interrater reliability of a modified Ashworth scale of muscle spasticity. Phys Ther. 1987;67:206-7.

31. Delp S, Anderson FC, Arnold AS, Loan P, Habib A, John CT, Guendelman E, Thelen DG. OpenSim: open-source software to create and analyze dynamic simulations of movement. IEEE Trans Biomed Eng. 2007;54:1940-50.

32. Van der Krogt MM, de Graaf WW, Farley CT, Moritz CT, Casius LJR, Bobbert MF. Robust passive dynamics of the musculoskeletal system compensate for unexpected surface changes during human hopping. J Appl Physiol. 2009; 107:801-80.

33. Thelen DG. Adjustment of Muscle Mechanics Model Parameters to Simulate Dynamic Contractions in Older Adults. J Biomech Eng. 2003;125:70.

34. Spasticity model in OpenSim [https://simtk.org/home/spasticitymodel]. Date accessed 27 May 2016.

35. Van der Helm FCT, Rozendaal L. Musculoskeletal systems with intrinsic and proprioceptive feedback. In: Winters JM, Crago PE, editors. Biomechanics and Neural Control of Posture and Movement. New York: Springer New York; 2000. p. 164-76.

36. Staude G, Wolf W. Objective motor response onset detection in surface myoelectric signals. Med Eng Phys. 1999;21:449-67.

37. Van der Krogt MM, Delp SL, Schwartz MH. How robust is human gait to muscle weakness? Gait Posture. 2012;36:113-9.

38. Arnold EM, Ward SR, Lieber RL, Delp SL. A model of the lower limb for analysis of human movement. Ann Biomed Eng. 2010;38:269-79.

39. Simulating the Effect of Contracture and Weakness on Walking Capacity in Cerebral Palsy [http://opensim.stanford.edu/support/event_details. html?id=123]. Date accessed 27 May 2016

40. Fee JW, Foulds RA. Neuromuscular modeling of spasticity in cerebral palsy. IEEE Trans Neural Syst Rehabil Eng. 2004;12:55-64.

41. Van Campen A, Pipeleers G, De Groote F, Jonkers I, De Schutter J. A new method for estimating subject-specific muscle-tendon parameters of the knee joint actuators: a simulation study. Int j numer method biomed eng. 2014;30:969-87.

42. De Bruin M, Smeulders MJ, Kreulen M, Huijing PA, Jaspers RT. Intramuscular connective tissue differences in spastic and control muscle: A mechanical and histological study. PLoS One. 2014;9:e101038.

43. Noble JJ, Fry NR, Lewis AP, Keevil SF, Gough M, Shortland AP. Lower limb muscle volumes in bilateral spastic cerebral palsy. Brain Dev. 2014;36:294-300.

44. Thilman A, Fellows S, Garms E. The mechanism of spastic muscle hypertonus. Variation in reflex gain over the time course of spasticity. Brain. 1991;114:233-44.

45. Lebiedowska MK, Fisk JR. Knee resistance during passive stretch in patients with hypertonia. J Neurosci Methods. 2009;179:323-30.

46. Pandyan AD, Price $\mathrm{Cl}$, Rodgers $\mathrm{H}$, Barnes MP, Johnson GR. Biomechanical examination of a commonly used measure of spasticity. Clin Biomech (Bristol, Avon). 2001;16:859-65.

47. Van den Noort JC, Scholtes VA, Becher JG, Harlaar J. Evaluation of the catch in spasticity assessment in children with cerebral palsy. Arch Phys Med Rehabil. 2010:91:615-23.

48. Gracies J-M. Pathophysiology of spastic paresis. II: Emergence of muscle overactivity. Muscle Nerve. 2005:31:552-71.

49. Kalsi G, Fry N, Shortland A. Dynamic measurement of gastrocnemius belly length during walking in typically developing children and children with cerebral palsy. Gait Posture. 2009;30:S18-9.

50. Gao F, Zhao H, Gaebler-Spira D, Zhang LQ. In vivo evaluations of morphologic changes of gastrocnemius muscle fascicles and achilles tendon in children with cerebral palsy. Am J Phys Med Rehabil. 2011;90:364-71.

51. Zhao H, Wu Y-N, Hwang M, Ren Y, Gao F, Gaebler-Spira D, Zhang L-Q. Changes of calf muscle-tendon biomechanical properties induced by passive-stretching and active-movement training in children with cerebral palsy. J Appl Physiol. 2011;111:435-42.

52. Scheys L, Desloovere K, Suetens P, Jonkers I. Level of subject-specific detail in musculoskeletal models affects hip moment arm length calculation during gait in pediatric subjects with increased femoral anteversion. J Biomech. 2011:44:1346-53.

53. Arnold AS, Blemker SS, Delp SL. Evaluation of a deformable musculoskeletal model for estimating muscle-tendon lengths during crouch gait. Ann Biomed Eng. 2001;29:263-74.

\section{Submit your next manuscript to BioMed Central and we will help you at every step:}

- We accept pre-submission inquiries

- Our selector tool helps you to find the most relevant journal

- We provide round the clock customer support

- Convenient online submission

- Thorough peer review

- Inclusion in PubMed and all major indexing services

- Maximum visibility for your research

Submit your manuscript at www.biomedcentral.com/submit

) Biomed Central 